\title{
ANALYZING MOBILE APPLICATION SOFTWARE POWER CONSUMPTION VIA MODEL-DRIVEN ENGINEERING
}

\author{
Chris Thompson, Douglas Schmidt \\ Dept. of Computer Science, Vanderbilt University, Nashville, TN, USA \\ $\{$ thomchr,schmidt $\} @$ dre.vanderbilt.edu \\ Hamilton Turner, Jules White \\ Dept. of Elec \& Computer Engineering, Blacksburg, VA, USA \\ $\{$ hturner,julesw\}@vt.edu
}

Keywords: mobile computing, low-power, model driven engineering

Abstract: $\quad$ Smartphones are mobile devices that travel with their owners and provide increasingly powerful services. The software implementing these services must conserve battery power since smartphones may operate for days without being recharged. It is hard, however, to design smartphone software that minimizes power consumption. For example, multiple layers of abstractions and middleware sit between an application and the hardware, which make it hard to predict the power consumption of a potential application design accurately. Application developers must therefore wait until after implementation (when changes are more expensive) to determine the power consumption characteristics of a design.

This paper provides three contributions to the study of applying model-driven engineering to analyze power consumption early in the lifecycle of smartphone applications. First, it presents a model-driven methodology for accurately emulating the power consumption of smartphone application architectures. Second, it describes the System Power Optimization Tool (SPOT), which is a model-driven tool that automates power consumption emulation code generation and simplifies analysis. Third, it empirically demonstrates how SPOT can estimate power consumption to within $\sim 3-4 \%$ of actual power consumption for representative smartphone applications.

\section{INTRODUCTION}

Emerging trends and challenges. Recent advances in mobile device and smartphone technologies have greatly increased the capabilities of these devices. For instance, the Google Nexus One has a $1 \mathrm{Ghz}$ processor and the Motorola Droid has a 550 Mhz processor, compared to the older Palm Treo's $315 \mathrm{Mhz}$ processor. Despite these performance increases, these devices still possess limited battery capacities that application developers must manage carefully.

To optimize power consumption effectively, developers must understand the trade-offs between performance and battery life, as well as the implications of their software architecture on power consumption. Functional requirements, such as minimum application response time, can conflict with power consumption optimization needs. For example, a traffic accident detection application (White et al., 2010) must be able to detect sudden accelerations indicative of a car accident. To detect acceleration events that in- dicate accidents, the application must sample device sensors and perform numerous calculations at a high rate. Conflicts occur between the functional requirements e.g. the minimum sensor sampling rate needed to accurately detect accidents, and the non-functional requirements e.g. sustaining operations on the mobile device without frequent battery recharging.

Due to complex middleware, OS, and networking layers, it is hard to predict the effects of application software architecture decisions on power consumption without actually implementing a design, which makes it hard to analyze the power consumption of design until late in the development cycle, when changes are more expensive (Kang et al., 2008). For example, a developer may elect to use HTTPS instead of HTTP to satisfy a security requirement by making communication between the application and server more confidential. It is currently hard, however, to predict how much additional power is consumed by the added encryption and decryption of data without actually implementing the system.

It is also hard to quantify the trade-off between 
power consumption and security, as well as many other design decisions. Moreover, certain design decisions, such as data transmission policies (e.g., should an application transmit immediately or wait for a specific medium like a $\mathrm{Wi}-\mathrm{Fi}$ or $3 \mathrm{G}$ cellular connection) are especially hard to analyze early in the design cycle, due to their variability. For instance, if an application only sends data intermittently, it may be beneficial to transmit small amounts of data over cellular connections due to the decreased power consumption of $3 \mathrm{G}$ cellular connection compared to $\mathrm{Wi}$ Fi (Agarwal et al., 2007). However, if a large amount of data must be sent, the total time required to transmit it over $3 \mathrm{G}$ may negate the benefit of using the less power consumptive connection. The cellular connection will take longer to transmit the data, therefore, which may in turn consume more total power than the Wi-Fi radio that can transmit the data faster.

Solution approach $\rightarrow$ Power consumption emulation of mobile software architectures with model-driven testing and auto-generated code. By using Model-driven Engineering (MDE) (Schmidt, 2006), we allow developers to specify a domainspecific modeling language(DSML) (Lédeczi et al., 2001) to capture key software architecture elements related to power consumption. Developers can then use automated code generators to produce emulation code from this model. Developers can also execute the generated emulation code on target hardware, collect power consumption information, and analyze the application's power consumption.

This emulation code allows developers to analyze a proposed software architecture prior to investing significant time and effort in a complete implementation of the design. The auto-generation of emulation code also enables developers to compare a range of different designs quantitatively during initial phases of a development process, which allows developers to select designs that satisfy both functional and non-functional requirements while minimizing power consumption. This analysis can also occur early in the software lifecycle (e.g., at design time), thereby avoiding costly changes being required later in order to optimize power consumption.

This paper describes the System Power Optimization Tool (SPOT), which uses MDE techniques to analyze the power consumption of mobile software architectures. SPOT allows developers to create highlevel models of candidate software architectures using the System Power Optimization Modeling Language (SPOML) that capture key software components related to power consumption. SPOT generates emulation code from the model that can be executed on target devices. As this emulation code is executed, it is also instrumented to collect power consumption data; the power data can later be downloaded and analyzed offline. This emulation and analysis cycle allows developers to understand the power consumption implications of their designs without expensive and time consuming manual programming using third-generation languages, such as $\mathrm{C}, \mathrm{C} / \mathrm{C}++$, and Java.

SPOT's generated emulation code mimics key power-consuming aspects of a proposed software architecture. Key power consumptive components of mobile application software architectures include GPS, acceleration, orientation, sensor data consumers, and network bandwidth consumers (Pering et al., 2006). Focusing SPOT on these components allows developers to model the most significant power expenditures of their applications. Moreover, as applications are constructed, the generated emulation code can be replaced incrementally with actual application components, allowing developers to refine the accuracy of the analysis continuously throughout the software lifecycle.

Paper organization. The remainder of this paper is organized as follows: Section 2 outlines a motivating example we use to showcase and evaluate SPOT's functionality throughout the paper; Section 3 summarizes the challenges associated with predicting power consumption of mobile application software architectures; Section 4 describes the structure and functionality of SPOT and SPOML; Section 5 empirically evaluates SPOT's power prediction capabilities and shows how its modeling primitives and emulation infrastructure can accurately predict power consumption for representative mobile applications on the Android smartphone platform; Section 6 compares SPOT with related work; and Section 7 presents concluding remarks.

\section{Motivating Example: the WreckWatch Case Study}

This section describes WreckWatch (White et al., 2010), which is an open-source ${ }^{1}$ mobile application we built on the Android smartphone platform to detect automobile accidents. We use WreckWatch as a case study throughout this paper to demonstrate key complexities of predicting the power consumption of mobile software architectures. As shown in Figure 1, WreckWatch operates by (1) monitoring smartphone sensors (such as GPS receivers and accelerom-

\footnotetext{
${ }^{1}$ WreckWatch is available from vuphone.googlecode.
} 


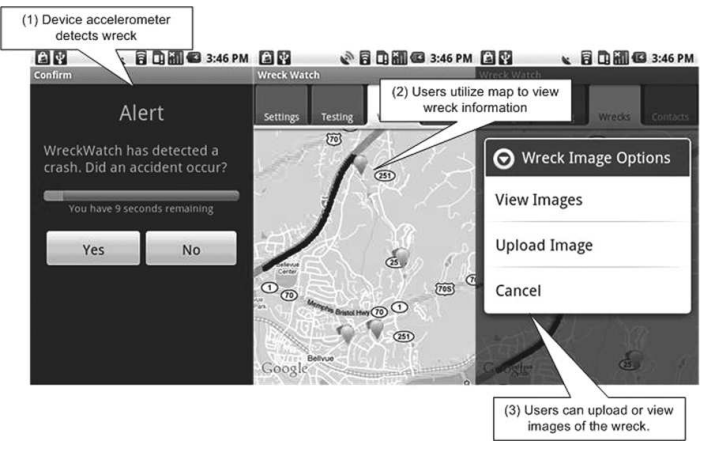

Figure 1: WreckWatch Operation

eters) for sudden acceleration/deceleration events that are indicative of an accident. Data about the event are then (2) uploaded to a remote server over HTTP where first-responders and/or other motorists can access the information via a web browser or Android client. WreckWatch allows bystanders (3) to upload images of the accident to the same web server, thereby increasing the information first-responders possess before arriving at the scene.

To detect traffic accidents accurately, WreckWatch runs continuously as a background service and continuously consumes a great deal of accelerometer and GPS data. The application must therefore be conservative in its power consumption. If not designed properly, WreckWatch can decrease smartphone battery life significantly.

\section{Challenges of Designing Power Conscious Mobile Applications}

This section describes the challenges associated with developing power-aware mobile software, such as the WreckWatch application described in Section 2. High-level mobile application development SDKs, such as Google Android or Apple iPhone, simplify mobile application development, but do not simplify power consumption prediction during application design. In fact, the abstractions present in these SDKs make it hard for developers to understand the power implications of software architecture decisions until their designs have been implemented (Parikh et al., 2002), as described in Section 3.1. Interaction with sensors, such as accelerometers or GPS receivers and network interaction, can also result in a significant amount of power consumption, as described in Sections 3.2 and 3.3.

\subsection{Challenge 1: Accurately Predicting Power Consumption of Framework API Calls}

Each line of code executed results in a specific amount of power consumed by the hardware. In the simplest case, this power consumption results from a small series of CPU operations, such as reading from memory or adding numbers. In some cases, however, a single line of code can result in a chain of hardware interactions, such as activation of the GPS receiver and increasing the rate at which the screen is redrawn. Moreover, although the higher levels of abstraction provided by modern smartphone SDKs make it easier for developers to implement mobile application software, they also complicate predictions of the effects on the hardware.

For example, WreckWatch heavily utilizes the Google Maps API and the "MyLocation" map overlay, which provides end users with a marker indicating their current GPS location. The use of the "MyLocation" is typically accomplished with fewer than 10 lines of code, but results in substantial power consumption. This is because the overlay is redrawn at a high rate to achieve a "flashing" effect, and because the overlay enables and heavily utilizes the GPS receiver on the device, which further increases power expenditure. It is hard to predict how using arbitrary API calls, such as this overlay, will affect application power consumption without implementing a particular design and testing it on a particular target device.

This abstraction in code makes powerconsumption analysis on arbitrary segments of code hard. Predicting power usage from high-level design abstractions, such as a UML diagram, is even harder. Section 4 describes the MDE and emulation approach we use to address this challenge.

\subsection{Challenge 2: Accurately Predicting Power Consumption of Sensor Usage Architectures}

In applications utilizing sensor data, the most accurate sensor data is obtained by sampling as often as possible. Sampling at high rates, however, incurs high power consumption (Krause et al., 2005) by not allowing sensors to enter low power modes and by increasing the amount of data processed by applications. Reducing the sample rate can decrease application power consumption considerably, but also reduces accuracy. The trade-offs between power consumption and accuracy at a given sampling rate are hard to determine without empirical tests on a target 
device due to the high-degree of layering in modern smartphone APIs and system architectures.

For example, WreckWatch was originally designed to collect GPS data every 500 milliseconds and consume accelerometer data at Android's predefined NORMAL rate setting. During the development of WreckWatch, it was clear that reducing WreckWatch's GPS sampling rate would reduce overall power consumption, but it was unclear to what degree. Moreover, it was hard to predict what sample rate would provide sufficient accuracy and still allow the phone to operate for days between charges. Section 4 describes how we use automatic code generation to create emulated applications that accurately analyze the power consumption of a candidate sensor utilization architecture without incurring the substantial time and effort to manually implement the architecture.

\subsection{Challenge 3: Accurately Assessing the Effects of Different Communication Protocols on Power Consumption Prior to Implementation}

Each application and network communication protocol has a specific overhead associated with it and can result in significant power consumption (Heinzelman et al., 2000). Certain protocols require more development overhead to implement, but have low runtime overhead (e.g. bandwidth consumption, message processing time, etc.). It is hard to determine early (e.g., at design time) in an applications lifecycle, however, how this overhead will affect power consumption and whether the number of messages transmitted will be substantial enough to impact power consumption significantly. This challenge is exacerbated if certain network operations consume more power than others, e.g., receiving data often consumes more power than transmitting data (Wang et al., 2006).

For example, to provide the most accurate situational awareness to first responders - and provide the most accurate congestion information to motoriststhe WreckWatch application must periodically request wreck information from the central web server. These updates must be done periodically and were originally intended to run over HTTP. Using HTTP results in a significantly less developer effort but results in a considerable amount of communication overhead from the underlying TCP and HTTP protocols, which ultimately transmits substantial amounts of data that have no relevance to the application. It is hard to determine at design time if/how this additional data transmission will significantly impact power consumption. Section 4 shows how we used MDE code generation to implement and analyze potential communication protocols rapidly.

\section{The System Power Optimization Tool (SPOT)}

This section describes the structure and functionality of the System Power Optimization Tool (SPOT), which is an MDE tool that allows developers to model potential mobile application software architectures to predict their power consumption, generate code to emulate that architecture, and then systematically analyze its power consumption properties. SPOT addresses the challenges described in Section 3 by allowing developers to understand the implications of their software architecture decisions at design time.

SPOT's development process enables developers to generate visual, high-level models rapidly, as shown in Figure 2. These models can then be used to analyze the power consumption of mobile application software architectures (step 1 of Figure 2). SPOT thus helps overcome key challenges of predicting power consumption by generating device logic that can be used to gather power consumption information on physical hardware during early phases of an application's software lifecycle, which helps minimize expensive redesign/refactoring costs in later phases.

SPOT uses models shown in Figure 2 to generate instrumented emulation code for the given platform or device (step 2 of Figure 2). When executed on actual hardware (step 3 of Figure 2), this generated code collects power consumption and system state information. This power consumption data can then be downloaded and analyzed offline to provide developers with application power utilization at key points throughout the lifecycle and execution path (step 4 of Figure 2).

SPOT also supports the use of custom code modules. These models allow developers to replace automatically generated code with actual application logic while still providing the same analytical capabilities available when using generated code. SPOT therefore not only allows developers to perform analysis early in the development cycle, but also to perform continuous integration testing throughout development.

SPOT is implemented as a plugin for the Eclipse IDE. Its runtime power consumption emulation and capture infrastructure is built using predefined, userconfigurable classes that emulate specific power consuming components, such as GPS data consumers. This infrastructure captures power consumption in- 


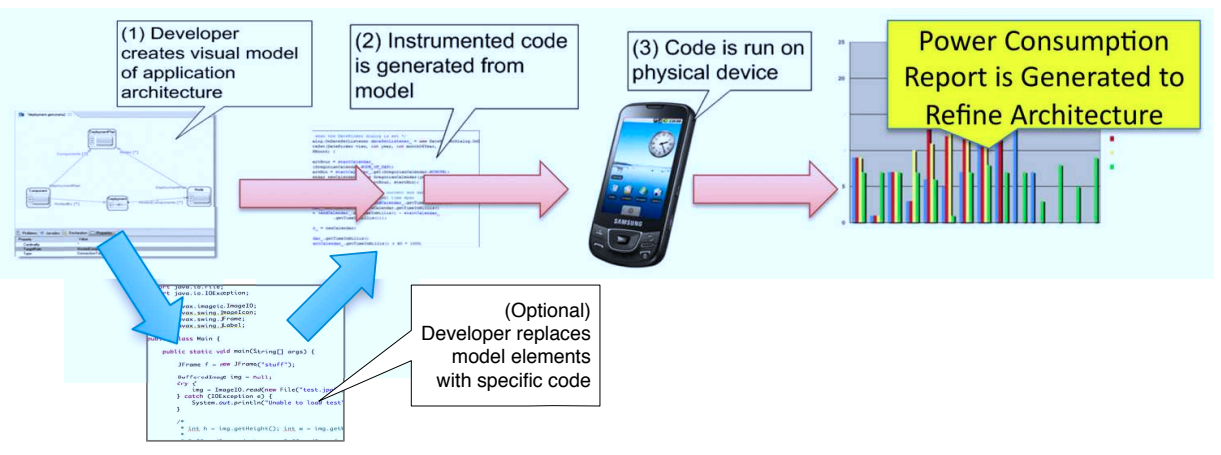

Figure 2: SPOT Modeling and Analysis Process

formation during executing by using the device's power API. For example, power data collection on the Android platform is performed by interfacing with the OS application power API, i.e., the Android power consumption API as implemented by the " $\mathrm{Fu}$ elGauge" application.

The remainder of this section describes how SPOT's DSML, emulation code generation, and performance measurement infrastructure help application developers address the challenges presented in Section 3. Section 4.1 describes SPOT's modeling language, SPOML, Section 4.2 describes how SPOT generates emulation code, and Section 4.3 describes how SPOT analyzes and evaluates emulation code during execution.

\subsection{Mobile Application Architecture Modeling and Power Consumption Estimation}

SPOT describes key power-consuming aspects of a mobile application via a DSML with specific language elements. This DSML allows developers to specify their software architecture visually with respect to power consuming components, as shown in Figure 2. Prior work (Thompson et al., 2009; White et al., 2010; Turner et al., ) showed how the following components are often significant power consumers in mobile applications:

- CPU consumers are used to represent CPUintensive code segments such as calculations on sensor data. Developers can specify the amount of CPU time that should be consumed by specifying the number of loop iterations of a square root calculation that should be run. For example, WreckWatch developers can model the mathematical calculation time to determine the current G-forces on the phone.

- Memory consumers generate dynamically allocated memory. These consumers allow developers to analyze not only the power consumed by actual operations, but also their impact (such as the frequency and duration of garbage collector sweeps) on garbage collection. Developers can specify the amount of memory to consume as bytes. For example, WreckWatch developers can model the effects of caching accident images of varying sizes.

- Accelerometer consumers, which interact with system accelerometers and consume accelerometer data. These consumers can be configured to utilize the full range of system-supported sample rates. For example, WreckWatch developers can model the sensor interaction needed to accurately detect car accidents.

- GPS consumers interact with the device's GPS receiver. These consumers can be configured with custom sample rates as well as a minimum distance between points, i.e., the sensor will only return a data point if the distance between the current point and the last point is greater than a specified value. GPS consumers allow developers to analyze the impact of using a location service configuration on power consumption. For example, WreckWatch developers use this capability to model how polling for a vehicle's location at different rates impacts power consumption.

- Network consumers emulate application network interaction by periodically transmitting and receiving data. Network consumers allow users to supply SPOT with sample data that is then transmitted at the interval specified. For example, WreckWatch developers can provide a URI along with server and port information to configure SPOT to make a specific request. These consumers can also be configured to execute at varying times to emulate periodic updates.

- Screen drawing agents utilize graphics libraries, such as OpenGL, to emulate a graphicsintensive application, such as a game or streaming video to a first responder's WreckWatch client. Users can configure these consumers by specifying the types and size of objects to draw on the screen, along with any transformations that should be performed on the object. For example, WreckWatch developers can use the drawing agents to show how the use of images and video for situational awareness impacts battery life.

- Custom code modules allow developers to 
specify their own code to run against the profiling package. This capability allows developers to extend SPOT's functionality to meet their needs, as well as incrementally replace the emulation code with actual application logic as it becomes available. Replacing the emulation logic allows developers to perform testing as development progresses and increase the accuracy of the evaluation and analysis. For example, WreckWatch developers can use these consumers to include a service for uploading multimedia content about an accident to a central web server.

The metamodel for SPOT's DSML, called the System Power Optimization Modeling Language (SPOML), allows application developers to build software architectural specifications that determine power consumption from key power consuming components. SPOML was created using the metamodeling features of the Generic Eclipse Modeling System (GEMS) (White et al., 2009), which is a tool for rapidly generating visual modeling tools atop the Eclipse Modeling Framework (EMF). GEMS is built atop Ecore, which provides metamodeling and modeling facilities for Eclipse (Budinsky et al., 2003).

The primary application serves as the root element of the model. Power consumption modules can exist within either activities (which are basic building block components for Android applications and represent a "screen" or "view" that provides a single, focused thing a user can do) or services (which are background processes that run without user intervention and do not terminate when an application is closed).

Each activity or service can contain one or more power consumer modeling elements described above. Developers can therefore emulate potential decisions that they make when designing a mobile device application, which allows them to emulate a wide range of application software architectures. For example, Figure 3 shows a SPOML model of the WreckWatch application's sensor usage design.

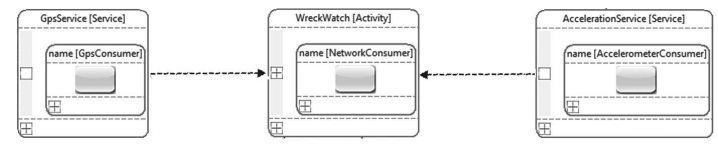

Figure 3: WreckWatch Model

Acceleration and GPS consumers run in independent services, while the network consumer runs in the application's activity. This model results in an application that monitors GPS and accelerometer values at all times regardless of what the user is doing. It only utilizes the network connection, however, when the user has the WreckWatch application open and running.

\subsection{Generating Software Architecture Emulation Code}

Predicting the power consumption of an arbitrary design decision is hard, as described in Section 3.1. SPOT addresses this challenge by generating application emulation code automatically to execute on the underlying device hardware. SPOT's automatic generation of emulation code allows application developers to reduce the time required to write enough code to analyze system power consumption accurately. This emulation code is instrumented so the architecture's power expenditures can be examined after a test run.

In addition to instrumenting the code, SPOT has the potential to apply the same model for multiple target platforms, such as Android and iPhone, as long as configurable code is available for the powerconsuming elements. This emulation and analysis cycle allows developers to observe the power consumption of their design very early in the development cycle, as well as evaluate their software designs across multiple hardware configurations to assess how changes in hardware affect application power consumption. For example, even though the Motorola Droid and Google Nexus One both run the Android platform, each possesses key hardware differences, such as the type and size of the display, that impact power consumption.

The generated emulation code allows developers to address the remaining challenges of selecting an optimal communication protocol and optimizing sensor polling rates, as described in Section 3. Generated emulation code allows developers to evaluate the power consumption of a potential design empirically, rather than simply guessing its power consumption or waiting until an application implementation is complete before running tests. Moreover, developers can quantitatively compare the power consumption effects of choosing different networking protocols and can evaluate the power consumption of different sensor sampling rates.

To accomplish this mobile software architectural emulation, SPOT uses a set of predefined code blocks that can be configured at runtime to perform power consuming operations. SPOT uses an XML configuration file to perform any necessary configuration and create an application that emulates the power consumption of the desired software architecture. To generate this XML configuration file, SPOT interprets the model outlined in Section 4.1. Users of SPOT define the model and tweak configuration parameters, and SPOT can then compile the model and parameters into an intermediate XML format which is utilized to configure prebuilt implementations of each 
power consuming element described in Section 4.1.

Figure 4 shows a sample of the XML configuration file generated for the WreckWatch model shown in Figure 3. The XML shown in Figure 4 represents

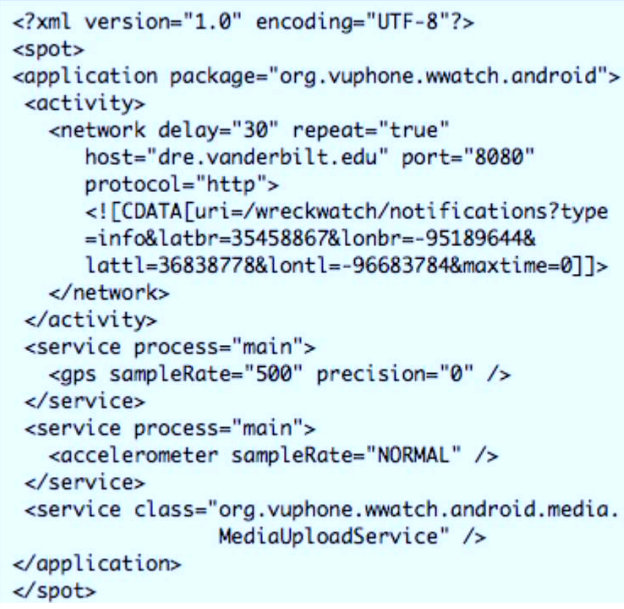

Figure 4: Sample WreckWatch Emulation XML

a configuration with two background services running an accelerometer consumer and a GPS consumer. The GPS consumer samples every 500 milliseconds, with a minimum radius between sample points set to 0 . The accelerometer consumer is set to sample at the NORMAL rate, which is a constant defined by Android. There is also a network consumer transmitting sample data every 30 seconds and repeating infinitely. The network consumer is configured to connect to a specific host on a specific port and utilize the HTTP protocol.

The predefined power consumers have configurable options (such as the sample rate of the GPS and data to transmit over the network) provided by the XML configuration file. These power consumption elements are generic and applicable to a wide range of platforms, such as Android, iPhone, or BlackBerry. The predefined power consumers are implemented on the Android platform as follows:

- CPU consumers are implemented as a set of nested loops that perform basic mathematical operations, such as square root calculations, on randomly generated data. This module utilizes the CPU while minimizing utilization of other system resources, such as memory that could skew power consumption information. For example, this consumer uses primitive types to avoid allocating dynamic memory. Users can adjust the length of the loops via a configurable parameter. Various end device processors result in same-length loops performing differently on divferent devices, in a manner similar to CPU-intensive al- gorithmic performance on end-user devices.

- Memory consumers are implemented by dynamically allocating custom objects that wrap byte arrays. To analyze the frequency of garbage collection, a Java WeakReference object is used to inform the garbage collector that they can be reclaimed, despite having active references within running code. The object's finalize () method (which is called immediately before the object is reclaimed by the Android Dalvik virtual machine) is overridden to record the time of garbage collection, thereby allowing developers to analyze the frequency of garbage collection runs. The WeakReference object will thus be reclaimed during every garbage collection run.

Due to the limitations of the Android instrumentation API, garbage collection and memory usage must be inferred through analysis of the frequency and duration of garbage collection runs, rather than through direct power consumption measurement. Although this limitation prevents developers from including memory statistics in the data along with CPU, sensor, and network utilization, they can still examine how their design uses memory. Users can also configure the amount of memory and frequency of allocation, as well as supply custom objects (such as WreckWatch's image caches) to use rather than the byte arrays used by default.

- GPS consumers are implemented by code that registers to receive location updates from the GPS receiver at specific intervals.

- Accelerometer consumers are implemented using the configuration specified in the XML file, along with generic setup code to establish a connection to the appropriate hardware.

- Network consumers are implemented as emulation code containing a timer that executes a given networking operation, such as an HTTP operation at a user defined interval.

- Screen drawing agents allow users to specify 3D and 2D graphics to draw on the screen. Developers will specify object contents along with any potential motion or actions.

- Custom code modules allow developers to supply their own code blocks to extend the functionality of SPOT and enhance emulation accuracy as the development cycle progresses by substituting the faux emulation code with actual application logic. SPOT allows developers to supply class files to load into the emulation application dynamically, as well as method "hooks" to allow the emulation code to interact with the custom code properly. 


\subsection{Power Consumption Instrumentation}

SPOT uses an instrumentation system to capture power consumption statistics as the emulation code is executed, as shown in Figure 5. Components in

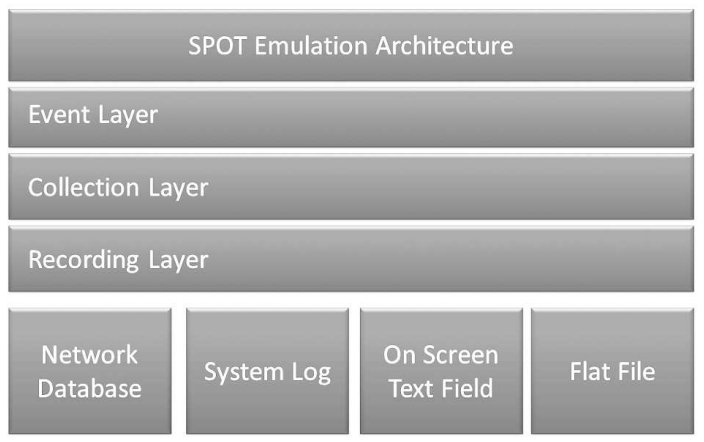

Figure 5: SPOT Instrumentation System

the instrumentation system are either Collectors, Recorders, or Event Handlers. Collectors interface directly with the specific power API on the system and pass collected data to Recorders, which persist the data collected by Collectors by writing it to a file or other storage medium. Event Handlers respond to the events fired by entering or leaving emulation code blocks.

These components are dynamically loaded via Java reflection to ensure extensibility and flexibility. For instance, developers can implement a custom Collector to monitor which components are in memory at any given time. Alternatively, developers could define Recorders to log power consumption information to another data storage medium, such as a local or network database rather than a flat file.

To analyze an architecture effectively, SPOT records battery state information over time to allow developers to pinpoint specific locations in their application's execution path that demand the most power. To collect power consumption information accurately, SPOT uses an event-driven architecture that precisely identifies the occurrence of each major application-state change, such as registering or unregistering a GPS listener and SPOT takes a "snapshot" of the power consumption when the application performs these operations. This event-driven architecture allows developers to understand the power consumption of the application before, during, and after key power-intensive components.

In addition to event-triggered power snapshots, SPOT also periodically collects power consumption information. This information allows developers to trace overall power consumption or power consumption within a given block. The power information Collector that collects snapshots and periodic samples can be configured to run in a separate process to prevent contamination of the data.

To accomplish event-driven power profiling, SPOT fires events immediately before an application enters a component that was defined in the model and immediately after it leaves a model-defined component. These events work in conjunction with the periodic power consumption updates to provide developers with a complete description of how their software architecture elements consume power. SPOT's eventdriven model of collecting power consumption data also allows developers to identify precisely what the application was doing when key power consumption spikes occur, further helping them optimize their designs.

SPOT's emulation infrastructure currently runs on the Android mobile platform and uses the power consumption API utilized by the "FuelGauge" application in the core Android installation. The power consumption API provides application developers with access to the amount of time the application utilizes the CPU, sensors, wake-locks, and other system resources, in addition to the overall power consumption.

Android's power consumption API provides power-consumption information on a per-package basis. By implementing SPOT in a different package, developers can analyze power consumption without influencing it. Collecting power consumption information in this manner increases accuracy. Moreover, SPOT can be implemented simply as a collector to analyze existing applications without modifying their source code.

\section{Results}

This section analyzes the results of experiments that empirically evaluate SPOT's MDE-based capabilities presented in Section 4. These experiments measure SPOT's ability to collect power consumption information on a given model, as well as accurately model the power consumption of a proposed application software architecture. These results show how SPOT can assess and analyze power consumption information gathered through the Android's power consumption API and evaluate SPOT's accuracy in predicting power consumption information about a software architecture at design time. 


\subsection{Hardware/Software Testbed}

All tests were performed on a Google Nexus One with a $1 \mathrm{Ghz}$ CPU, $512 \mathrm{MB}$ of RAM, 512MB of ROM and a 4 GB SD card running the default installation of Android 2.1 (Eclair). The SPOT application was the only third-party application running at the time of experimentation. The same power consumption information gathering logic was used to collect information on emulation code, as well as the sample applications. The information was analyzed in Excel based on power consumption data written to the device's SD card in the form of a CSV file.

To assess the consumption characteristics of different designs, the current SPOT version generates an Android application package. It then periodically samples the battery usage statistics from the OS writing these values to a CSV file on the SD card. SPOT also fires events when the application's state changes, e.g., when the GPS is started or a sensor is disconnected. These events allow SPOT users to examine the power consumption of active hardware, in addition to the overall consumption of the application.

SPOT uses an XML-based configuration file that is generated from the SPOML model described in Section 4.1. This XML file is loaded on to the device's SD card and parsed at startup. Due to the way that the power consumption API collects information, the data gathered reflects only power consumed by the SPOT application and does not include any power consumed by system processes, such as the GUI display or garbage collector.

\subsection{Experiment 1: Empirical Evaluation of SPOT's Emulation Code Accuracy}

Overview. This experiment quantitatively compares the power consumption of two Android applications and the power consumption of the emulation code derived from their respective SPOT models. Ensuring SPOT's accuracy is important since it allows developers to compare the power consumption of key power consuming components in their mobile architecture.

The applications used in this experiment are the WreckWatch application presented in Section 2 and OpenGPSTracker (open-gpstracker. googlecode.com), which is an open-source Android application that uses GPS to track the coordinates of the phone and display it on a Google Map on the device. The GPS points, and other information about the route, are stored on the device to allow the user to replay the route later. OpenGPSTracker also determines device speed as GPS points are collected.

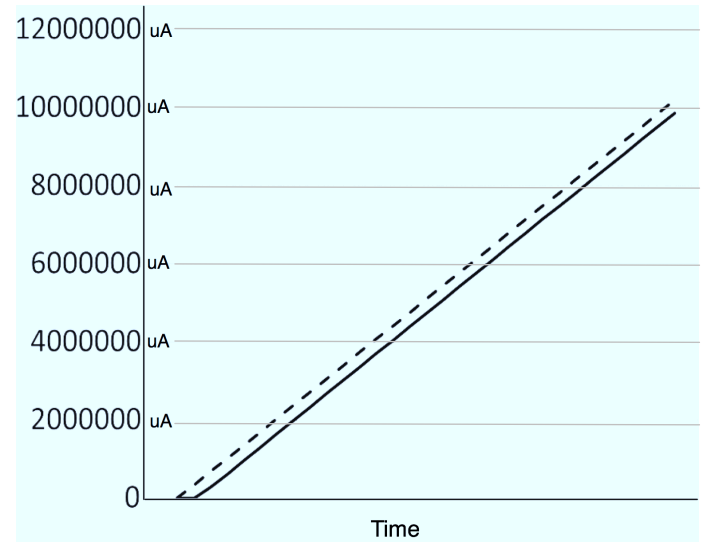

Figure 6: Comparison of WreckWatch Application Logic and Emulation Code

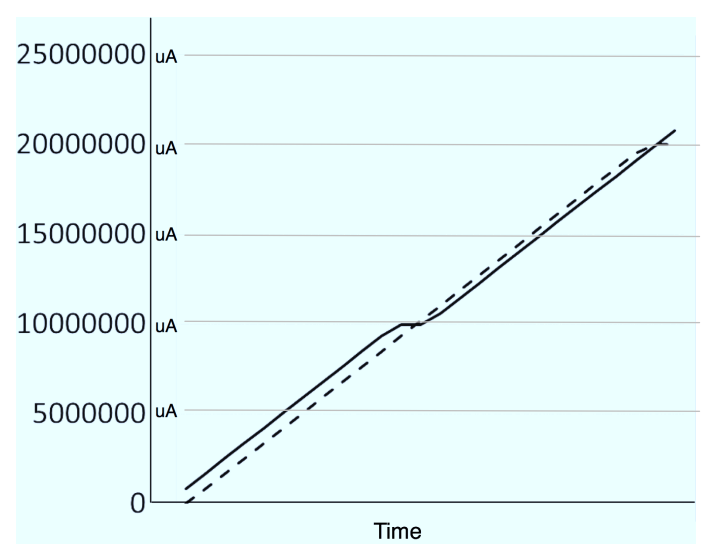

Figure 7: Comparison of OpenGPSTracker Application Logic and Emulation Code

Hypothesis. SPOT is intended to provide developers with an estimate of how a proposed application software architecture will consume power. We therefore hypothesized that SPOT could provide power consumption information to within $25 \%$ of the actual power consumption of WreckWatch and OpenGPSTracker. Based on prior work (Thompson et al., 2009; White et al., 2010; Turner et al., ), we also hypothesized that the components we chose represented the key factors in mobile application power consumption and would be adequate to provide this level of accuracy.

WreckWatch results. Figure 6 shows the graph of the actual power consumption of the WreckWatch application compared with the power consumption of the WreckWatch emulation code generated by SPOT. The emulation code's power consumption follows the same trend as that of the application and provided a final power consumption value that was within $3 \%$ 
of the actual power consumed by WreckWatch. The SPOT model consisted solely of GPS and accelerometer consumers and did not attempt to model any additional aspects of the WreckWatch application. The model was accurate due to the substantial amount of power required by the GPS receiver. This result confirms that the GPS, sensor, and network modules that SPOT provides are key determinants of mobile power consumption. Although WreckWatch performs a number of CPU-intensive calculations on sensor data to determine if an accident occurred, these calculations are minor power consumers compared to sensor users.

OpenGPSTracker results. Figure 7 shows the graph of the actual power consumption of the OpenGPSTracker application compared with the power consumption of the emulation code generated by SPOT. As with the WreckWatch emulation code, the OpenGPSTracker emulation code consumes power at a rate similar to the actual application. Over the same time period, the emulation code for the OpenGPSTracker application was accurate at predicting power consumption to within 4\%. The SPOT model for the OpenGPSTracker application only used a GPS consumer and did not attempt to model any Google Maps functionality (or requisite network functionality) or any processing required to determine speed or store the location information. In this instance, the GPS power consumption was sufficient to model the power consumption of the entire application.

With both applications, SPOT modeled and predicted the power consumption of the actual application to within $4 \%$ of the actual power consumption. This result confirms our hypothesis that SPOT can provide an accurate prediction of power consumption by modeling key components of a mobile application.

\subsection{Experiment 2: Qualitative Comparison of Sensor Sample Rates}

Overview. This experiment evaluates the effects of sensor sample rates on an application's overall power consumption. The rate at which sensor data is consumed can have a significant impact on application power consumption, as described in Section 3.2. For example, Android's accelerometer provides four enumerations for sample rate: NORMAL, UI, GAME, and FASTEST. Although these sample rates provide varying levels of QoS, the trade-off between a given level of QoS and power consumption is not readily apparent at design time. The enumeration names give developers a clue to potential uses, as well as rank these sample rates according to rate and consequently power consumption. Alternatively, the GPS receiver allows developers to specify a value as the delay, in milliseconds, between samples.

SPOT allows developers to evaluate the power consumption of potential sensor sample rates. For experiment 2, we compared the power consumption of the GPS receiver while sampling at once every 2 seconds, once every 30 seconds, and once every 500 milliseconds.

Hypothesis. We hypothesized that SPOT could capture the power consumption effects of sensor sampling rate changes. In particular, we believed sampling rate changes of a few hundred milliseconds would produce power consumption changes that SPOT could detect.

Results. Figure 8 show SPOT's output for three different sample rates for the GPS sensor. The dashed

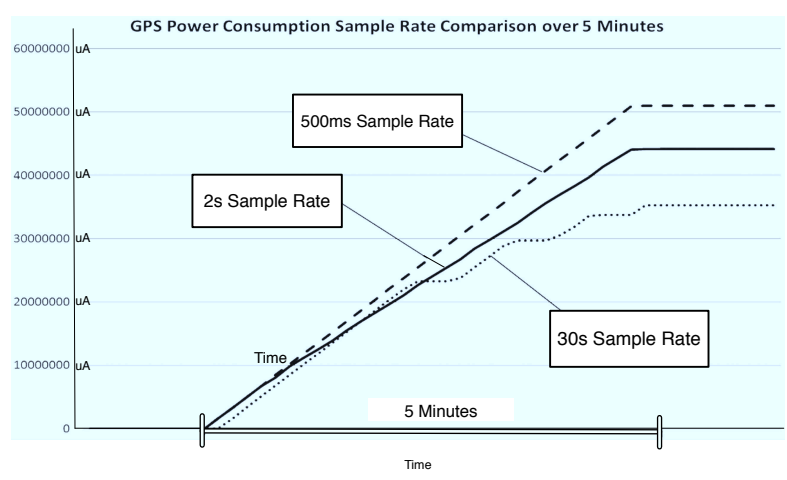

Figure 8: GPS Sample Rate Comparison

line represents the power consumption of the application when the sensor was sampled every 500 milliseconds, the solid line represents a sample taken every 2 seconds, and the dotted line represents the power consumption of the application sampling the sensor twice per minute. The samples in this graph were collected over 5 minutes and support the following observations:

- Power consumption during the first several seconds is uniform regardless of sample rate. Each graph is approximately equivalent for the first several seconds of data gathered during the GPS sampling, which implies that if developers need access to GPS for a short period of time, the greatest benefit would come from using a higher sample rate.

- The greatest improvement in power consumption due to a lower sample rate will occur over time. Although the graphs demonstrate a noticeable difference in power consumption over the 5-minute sample period, the improvement in battery life from a change in sample rate will only be realized when sampling occurs over an extended period of time. 
Ultimately, the amount of power consumed by the GPS receiver is directly proportional to the sampling rate of the application. Reducing the sampling rate of the GPS receiver is an effective means to reduce the overall power consumption of an application if the receiver is active for longer than $\sim 2$ minutes.

\subsection{Summary and Analysis of Results}

The results of the two experiments presented above show how SPOT can accurately analyze and predict an application's power consumption based on a model of the application software architecture. This capability allows developers to understand the implications of their design decisions early in the software lifecycle, i.e., without implementing the complete application. The emulation code SPOT generates is accurate, e.g., for our WreckWatch application it could predict power consumption within $3 \%$ of the actual application's power consumption. SPOT's accuracy stems in part from the significant power consumption of hardware components, such as the GPS receiver, that consume significantly more power than other hardware components on the device, such as the CPU or even accelerometers.

\section{Related Work}

This section describes previous work in five categories: (1) system execution modeling tools, (2) hardware-based power consumption optimization, (3) network protocol and interface optimization, (4) postimplementation power consumption analysis, and (5) our previous work on design-time power consumption analysis.

System execution modeling tools. The Component Utilization Test Suite (CUTS) (Hill et al., 2008) is a system execution modeling tool (Smith and Williams, 2001) that allows developers to model key architectural elements of distributed systems that determine performance. CUTS allows developers to model a distributed software architecture and then generate emulation code to predict performance. Although CUTS and SPOT share common domainspecific modeling patterns, CUTS's modeling abstractions focus on performance-related aspects of distributed systems, whereas SPOT's modeling abstractions focus on important power consumers in mobile applications, such as GPS usage. Moreover, it is not possible to capture power consumption information from CUTS emulation code or to generate mobile emulation applications for Android or iPhone.
Hardware-based power optimizations. Conventional techniques for reducing mobile device power consumption have focused on hardware- or firmwarelevel modifications to achieve the target consumption (Pering et al., 2006). These techniques are highly effective, but are limited to environments in which the hardware can be modified by the end user or developer. Further, these modifications tend to result in a tightly coupled architecture which makes them difficult to use outside of the context in which they were developed. In other words, a solution might reduce power consumption for one application or in one environment, but may not have the same effect if any of those assumptions change. Moreover, such modifications are typically beyond the scope of software engineering projects and require substantial understanding of low-level systems. In some instances, hardwarelevel modifications can actually hurt power consumption by increasing overhead. These techniques are also useful for reducing overall power consumption but do not help in power consumption analysis that is necessary when developing power-conscious applications. SPOT is complimentary to these approaches in that developers can use SPOT to identify the most effective method to minimize power consumption without requiring extensive hardware knowledge or restricting the optimizations a single hardware platform.

Network protocol and interface optimization. Due to the limited battery power available to mobile and embedded systems, a great deal of work has been done on the notion of optimizing system power consumption. Network interfaces consume a large portion of overall device power (Krashinsky and Balakrishnan, 2005) and consequently, a great deal of work has focused on reducing the power consumption of networking components. Ultimately, the amount of power consumed is directly proportional to the amount of data transmitted (Feeney and Nilsson, 2001) and in some instances require 100 times the power consumed by one CPU instruction to transmit one byte of data (Liu et al., 2004). Therefore, the power consumption of the network interface can be reduced by reducing the amount of data transmitted. Moreover, utilizing MAC protocols that reduce contention can significantly reduce power consumption (Chen et al., 1998). While MAC-layer modification is effective, it is typically beyond the scope of software-engineering projects, which is common with mobile application development.

SPOT seeks to accomplish similar goals by modifying the data transmitted by the application layer, rather than attempting to modify the underlying network stack. SPOT helps developers analyze the data they transmit to maximize throughput of relevant data 
(e.g. actual data versus markup or framing overhead) thereby reducing power consumption. In addition, SPOT can function in a complimentary role allowing developers to analyze the power consumption of network protocol optimizations to identify the most effective configuration.

Post-implementation power consumption analysis. Previous work (Creus and Kuulusa, 2007) not only identified software as a key player in mobile device power consumption, specifically Symbian devices, but also sought to allow developers to analyze the power consumption of applications during execution. Moreover, other work (Landsiedel et al., 2005) utilized a similar approach to analyze the power consumption of sensor nodes in a wireless sensor network. This power consumption analysis provided highly accurate results but suffers from the pitfalls of post-implementation testing, including increased cost of refactoring if problems are discovered. To prevent costly post-implementation surprises, SPOT allows developers to analyze designs before any code is written and allows them to perform continuous integration testing through the use of custom code components to further refine the accuracy of the model as development progresses.

This paper also extends our earlier work on MDE power consumption described in (Thompson et al., 2009) by providing the following new significant contributions: (1) it quantitatively examines SPOT's accuracy in evaluating power consumption of software architectures, (2) it examines the impact of different software architecture components, such as GPS utilization, on overall application power consumption, (3) it empirically evaluates the effects of software architecture design decisions, such as sensor sample rate, on overall application power consumption, and (4) it describes how data produced by SPOT can be utilized to refine and optimize the power consumption of a sample application.

\section{Concluding Remarks}

The System Power Optimization Tool (SPOT) is an MDE tool that allows developers to evaluate the power consumption of potential mobile application architectures early in the software lifecycle, e.g., at design time. Our experiments indicate that SPOT provides a high degree of accuracy, e.g., it predicted the power consumption of the WreckWatch and OpenGPSTracker applications to within $\sim 3-4 \%$. We learned the following lessons developing and evaluating SPOT:

- Sensor sample rates play an important role in long-term power consumption. The power con- sumed by the device sensors is largely uniform over the first several minutes of activation regardless of sample rate. It is only when these sensors are used for an extended period that the benefit of lower sample rates is realized. Developers must therefore consider the amount of time to activate the sensor in addition to the overall sample rate.

- Certain hardware components draw significantly more power than others. In the case of utilizing the GPS receiver, the power consumed by the location (GPS) service is so substantial that it becomes difficult to identify the effects of enabling or disabling other hardware components. Due to this "masking" effect, developers may overlook a significant consumer of power in an application. In general, small changes in GPS sample rate can have significant impacts on overall application power consumption.

- Certain system-related operations such as garbage collection are not reflected in data gathered by SPOT. Through the current method of data collection SPOT is only able to gather power consumption information about operations that it performs such as CPU, memory or sensor operations that it specifically requests. Our future work will therefore develop mechanisms for capturing the impact of these services on power consumption.

- Power consumption of networking hardware is dependent on data transmitted which is often dependent on user interaction. The power consumption of hardware, such as the Bluetooth or Wi-Fi interfaces, is dependent on the data transmitted and received on the device. This data is often generated at run-time by user interaction with the program. While it is effective for the developer to generate some sample data and provide it to SPOT, it would be more effective if developers could simply describe the data, e.g., via a schema file. Our future work is extending SPOT so it can process a data schema file and generate data that is random, yet still valid to the application.

- Although GPS is a major consumer of power, not all applications rely on GPS. Although GPS is a major consumer of power on today's smartphone devices, it is still important to understand the power consumption of applications that do not use the GPS, even if their power consumption is less drastic. Our future work is therefore analyzing SPOT's accuracy with mobile applications (such as 3D games with acceleration-based controls, streaming video players, and audio recording/processing applications) that do not use GPS, such as 3D games, feed readers, and multimedia applications.

SPOT is available in open-source form at syspower.googlecode. com. 


\section{REFERENCES}

Agarwal, Y., Chandra, R., Wolman, A., Bahl, P., Chin, K., and Gupta, R. (2007). Wireless wakeups revisited: energy management for voip over wi-fi smartphones. In ACM MobiSys, volume 7.

Budinsky, F., Steinberg, D., Merks, E., Ellersick, R., and Grose, T. J. (2003). Eclipse Modeling Framework. Addison-Wesley, Reading, MA.

Chen, J., Sivalingam, K., Agrawal, P., and Kishore, S. (1998). A comparison of MAC protocols for wireless local networks based on battery power consumption. In IEEE INFOCOM, volume 1, pages 150-157. Citeseer.

Creus, G. and Kuulusa, M. (2007). Optimizing Mobile Software with Built-in Power Profiling. Mobile Phone Programming and its Application to Wireless Networking, F. Fitzek and F. Reichert, Eds. Springer.

Feeney, L. and Nilsson, M. (2001). Investigating the energy consumption of a wireless network interface in an ad hoc networking environment. In IEEE INFOCOM, volume 3, pages 1548-1557. Citeseer.

Heinzelman, W., Chandrakasan, A., and Balakrishnan, H. (2000). Energy-efficient communication protocol for wireless microsensor networks. In Proceedings of the 33rd Hawaii International Conference on System Sciences, volume 8, page 8020. Citeseer.

Hill, J., Schmidt, D. C., Slaby, J., and Porter, A. (2008). CiCUTS: Combining System Execution Modeling Tools with Continuous Integration Environments. In Proceeedings of 15th Annual IEEE International Conference and Workshops on the Engineering of Computer Based Systems (ECBS), Belfast, Northern Ireland.

Kang, J., Park, C., Seo, S., Choi, M., and Hong, J. (2008). User-centric prediction for battery lifetime of mobile devices. In Proceedings of the 11th Asia-Pacific Symposium on Network Operations and Management: Challenges for Next Generation Network Operations and Service Management, pages 531-534. Springer.

Krashinsky, R. and Balakrishnan, H. (2005). Minimizing energy for wireless web access with bounded slowdown. Wireless Networks, 11(1):135-148.

Krause, A., Ihmig, M., Rankin, E., Leong, D., Gupta, S., Siewiorek, D., Smailagic, A., Deisher, M., and Sengupta, U. (2005). Trading off prediction accuracy and power consumption for context-aware wearable computing. In Proceedings of the Ninth IEEE International Symposium on Wearable Computers, pages 2026. IEEE Computer Society.

Landsiedel, O., Wehrle, K., and Gotz, S. (2005). Accurate prediction of power consumption in sensor networks. In Proceedings of The Second IEEE Workshop on Embedded Networked Sensors (EmNetS-II). Citeseer.

Lédeczi, Á., Bakay, A., Maroti, M., V

"olgyesi, P., Nordstrom, G., Sprinkle, J., and Karsai, G. (2001). Composing domain-specific design environments. Computer, pages 44-51.
Liu, T., Sadler, C., Zhang, P., and Martonosi, M. (2004). Implementing software on resource-constrained mobile sensors: experiences with impala and zebranet. In Proceedings of the 2nd international conference on Mobile systems, applications, and services, pages 256-269. ACM New York, NY, USA.

Parikh, D., Skadron, K., Zhang, Y., Barcella, M., and Stan, M. (2002). Power issues related to branch prediction. In Proceedings of the Eighth International Symposium on High-Performance Computer Architecture, pages 233-44. Citeseer.

Pering, T., Agarwal, Y., Gupta, R., and Want, R. (2006). Coolspots: Reducing the power consumption of wireless mobile devices with multiple radio interfaces. In Proceedings of the 4th International Conference on Mobile systems, Applications and Services, page 232. ACM.

Schmidt, D. C. (2006). Model-Driven Engineering. IEEE Computer, 39(2):25-31.

Smith, C. and Williams, L. (2001). Performance Solutions: A Practical Guide to Creating Responsive, Scalable Software. Addison-Wesley Professional, Boston, MA, USA.

Thompson, C., White, J., Dougherty, B., and Schmidt, D. (2009). Optimizing Mobile Application Performance with Model-Driven Engineering. In Proceedings of the 7th IFIP Workshop on Software Technologies for Future Embedded and Ubiquitous Systems.

Turner, H., White, J., Thompson, C., Zienkiewicz, K., Campbell, S., and Schmidt, D. Building Mobile Sensor Networks Using Smartphones and Web Services: Ramifications and Development Challenges.

Wang, Q., Hempstead, M., and Yang, W. (2006). A realistic power consumption model for wireless sensor network devices. In Proceedings of the Third Annual IEEE Communications Society Conference on Sensor, Mesh and Ad Hoc Communications and Networks (SECON).

White, J., Clarke, S., Dougherty, B., Thompson, C., and Schmidt, D. (2010). R\&D Challenges and Solutions for Mobile Cyber-Physical Applications and Supporting Internet Services. Springer Journal of Internet Services and Applications (to appear).

White, J., Hill, J., Tambe, S., Gray, J., Gokhale, A., and Schmidt, D. C. (2009). Improving Domain-specific Language Reuse through Software Product-line Configuration Techniques. IEEE Software Special Issue: Domain-Specific Languages and Modeling. 